\title{
Computation Rate Maximization for Wireless Powered Mobile Edge Computing with NOMA
}

\author{
Ming Zeng, Rong Du, Viktoria Fodor and Carlo Fischione \\ School of Electrical Engineering and Computer Science \\ KTH Royal Institute of Technology, Stockholm, Sweden \\ \{mzeng, rongd, vfodor, carlofi\}@kth.se
}

\begin{abstract}
In this paper, we consider a mobile edge computing (MEC) network, that is wirelessly powered. Each user harvests wireless energy and follows a binary computation offloading policy, i.e., it either executes the task locally or offloads it to the MEC as a whole. For the offloading users, non-orthogonal multiple access (NOMA) is adopted for information transmission. We consider rate-adaptive computational tasks and aim at maximizing the sum computation rate of all users by jointly optimizing the individual computing mode selection (local computing or offloading), the time allocations for energy transfer and for information transmission, together with the local computing speed or the transmission power level. The major difficulty of the rate maximization problem lies in the combinatorial nature of the multiuser computing mode selection and its involved coupling with the time allocation. We also study the case where the offloading users adopt time division multiple access (TDMA) as a benchmark, and derive the optimal time sharing among the users. We show that the maximum achievable rate is the same for the TDMA and the NOMA system, and in the case of NOMA it is independent from the decoding order, which can be exploited to improve system fairness. To maximize the sum computation rate, for the mode selection we propose a greedy solution based on the wireless channel gains, combined with the optimal allocation of energy transfer time. Numerical results show that the proposed solution maximizes the computation rate in homogeneous networks, and binary offloading leads to significant gains. Moreover, NOMA increases the fairness of rate distribution among the users significantly, when compared with TDMA.
\end{abstract}

\section{INTRODUCTION}

Recently, radio frequency (RF) based wireless power transfer (WPT) has drawn considerable attention owing to its potential for addressing the finite battery capacity problem of Internet of Thing applications [1]-[3]. In a WPT assisted network, the access point (AP) first transmits RF energy to the users over the downlink. The users then exploit the harvested energy to maintain local operations, such as sensing, computation and actuation, or to transmit information over the uplink, and utilize cloud or edge computing for the information processing.

Early works in WPT assisted networks adopt time division multiple access (TDMA) for uplink, and show that there exists a double near-far effect [2], [3]. This means that the user at the cell-edge not only receives much less energy from the $\mathrm{AP}$, but also has to transmit at higher power to achieve the same rate as the cell-center users. As a result, the achievable rates at the cell-edge users are much lower than those of the cell-center users, yielding substantial system unfairness. To address unfairness, non-orthogonal multiple access (NOMA) has been proposed for uplink in WPT assisted networks [4][7]. In [4], both sum throughput maximization and max-min rate maximization have been considered. In [5], the authors address the optimization of the RF power of the AP to increase the long-term weighted sum rate. The authors in [6] prove that the achievable sum throughput is independent from the decoding order, and proposes to improve the system fairness by applying a sequence of different decoding orders within a transmission interval. The work in [7] considers the sum rate maximization by jointly designing the time allocation for energy transfer, the downlink energy beamforming, and the receiver beamforming for a multi-antenna NOMA network.

An interesting natural question is how to use WPT with the technology of mobile edge computing (MEC), which is emerging as an effective solution to enhance the computing capability of wireless devices [8], [9]. In MEC, users offload their computationally intensive tasks to nearby servers located at the edge of radio access network, e.g., base stations (BSs) and WiFi APs. Compared with conventional cloud computing, latencies and jitter can be significantly reduced in MEC as the computing resources are located in proximity of the mobile devices. Moreover, with the advancement of hardware, the edge servers can own computation capacity comparable with that of a computer server a decade ago. This enables MEC to become one of the most promising technologies to support latency-critical and computation-intensive applications.

WPT assisted MEC has been considered in [10]-[13]. Specifically, reference [10] considers a single-user with binary offloading, aiming to maximize the probability of successful computation. The work in [11] studies a multi-user scenario, where a multi-antenna AP is deployed to charge the users. Optimal resource allocation is developed to minimize the AP's total energy consumption under the users' individual latency constraints. In contrast, both [12] and [13] investigate the problem of the maximization of the sum computation rate of a WPT assisted MEC network. The authors of [12] and [13] assume TDMA for the uplink, and adopt partial and binary offloading policy, respectively. The main challenges addressed in these works are the calculation of optimal TDMA slot length, and the selection of the uploading users. Specifically, [13] acknowledges that optimal binary uploading seems to follow a threshold based policy in homogeneous systems, where the energy harvesting and computational capabilities of the users are identical. 


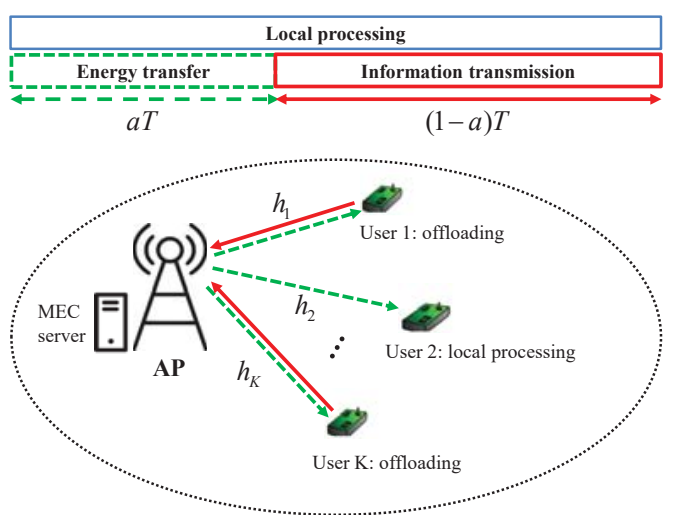

Fig. 1: System model.

In this paper, we consider multi-user WPT assisted MEC networks. The users can process the computing task locally or offload it to the MEC server. We investigate the sum computation rate maximization problem for all the users. For the offloading users, NOMA is adopted for uplink transmission [14], [15]. Compared to [4]-[7], this problem is more challenging since a binary offloading decision needs to be considered for each user. Compared to [10]-[13], the use of NOMA does not change the complexity of the resource allocation problem, while it lowers the synchronization requirement and reduces the system's signal overhead [6]. NOMA is also expected to improve the system fairness if appropriate decoding order is used.

We show that under a given mode selection, the energy transfer time allocation problem is concave and thus the optimal allocation can be found using bisection search. Then, we propose a greedy solution based on channel gains for mode selection. We derive closed-form expressions for the optimal uplink transfer times for TDMA and prove that NOMA achieves the same sum computation rate. Moreover, we show that this maximum achievable sum rate is independent from the decoding order of NOMA. We discuss the performance of WPT assisted MECs based on numerical evaluations. We demonstrate that our greedy offloading decisions achieve optimal performance in the considered scenarios, and deliver significant gains compared to systems applying local computing or offloading only. We show as well that NOMA can indeed improve the fairness compared to the TDMA counterpart.

The rest of the paper is organized as follows. Section II introduces the system model and the problem formulation. Section III presents the proposed NOMA based solution and Section IV discusses the TDMA baseline scheme. We show the system performance in Section V. Finally, we conclude the paper in Section VI.

\section{System Model And Problem Formulation}

\section{A. System Model}

As shown in Fig. 1, we consider a WPT assisted MEC network with a single-antenna AP and multiple single-antenna users. We denote the set of users by $\mathcal{K},|\mathcal{K}|=K$. The AP is integrated with a RF energy transmitter and a MEC server, while each user is equipped with an energy harvesting circuit and a rechargeable battery that can store the harvested energy to power its operations. Meanwhile, both the AP and the users have a communication circuit. It is assumed that WPT and communication are performed over the same frequency band. To avoid interference, time-division is adopted, i.e., the AP first broadcasts the RF energy to charge the users, and then, the users exploit the harvested energy to power their operations.

We consider the scenario where each user needs to run a certain computing task within each time frame $T$ using locally acquired data. For example, a user could be a wireless sensor, which should regularly generate an estimate of pollution level according to the raw data samples measured from the environment [13]. In each time frame, each user performs the computing locally using the on-chip micro-processor, or offloads the data to the MEC server. Specifically, we consider binary computation offloading, that is, a user operates either in the local computing mode, or in the offloading mode. The former requires no communication, but the computing capacity is limited due to the simple hardware and the limited harvested energy. In contrast, the latter is not constrained by computing capability, but the limited available energy, which may allow only the transmission of data having low resolution.

\section{B. Computation Model}

Denote the channel gain between the AP and user $i$ by $h_{i}$, which is assumed to be constant within a time frame of $T$, but may vary across different time frames. Besides, $h_{i}$ is considered the same for both downlink and uplink due to channel reciprocity.

Denote the time for WPT in time frame $T$ as $a T, a \in[0,1]$. Then, the energy harvested by user $i$ is given by

$$
E_{\mathrm{H}, i}=\mu P h_{i} a T,
$$

where $P$ is the transmit power of the AP, while $\mu \in(0,1)$ denotes the energy harvesting efficiency of the user. User $i$ will use the harvested energy to perform the computation task. It can either offload the task to the MEC or perform the task locally. Depending on its mode selection, the detailed operation is as follows.

1) Local Computing: As the energy harvesting circuit and the computing unit are separate, a local computing user can harvest energy and compute its task simultaneously [11]. This means that the entire time frame of $T$ can be used for computing. We denote the number of computation cycles required for processing one bit of raw data as $\phi_{i}$, which depends on the nature of the task at user $i$. Let $f_{i}$ be the processor's chosen computing speed (cycles/s), satisfying $f_{i} \leq f_{\max }$, where $f_{\max }$ is the maximum computation speed. Let $\Gamma_{i}$ denote the computation energy efficiency coefficient of the processor. The power consumption of the processor can be modeled as $\Gamma_{i} f_{i}^{3}$ (Joule/s) [16]. We represent the computation time of user $i$ by $t_{i}$, satisfying $0 \leq t_{i} \leq T$. Then, the energy consumption is $E_{\mathrm{L}, i}=\Gamma_{i} f_{i}^{3} t_{i}$, while the computation rate, i.e., the amount of bits processed in the time frame is given by

$$
r_{\mathrm{L}, i}=\frac{f_{i} t_{i}}{\phi_{i} T} \text {. }
$$


To ensure a sustainable operation at user $i$, the consumed energy should not exceed that of the harvested energy, i.e., $E_{\mathrm{L}, i} \leq E_{\mathrm{H}, i}$. Meanwhile, it is assumed that a user can always drain the harvested energy within $T$ if operating at the maximum computing speed, i.e.,

$$
E_{\mathrm{H}, i}=\mu P h_{i} a T \leq \mu P h_{i} T<\Gamma_{i} f_{\max }^{3} T
$$

holds for any practical value of $h_{i}$.

2) Offloading: Since time division is adopted for the energy transfer, an offloading user can only offload its task to the AP after energy harvesting, i.e., $(1-a) T$ is used for offloading. As in [10]-[13], we neglect the time spent on task computation at the AP and on result feedback, and thus, $(1-a) T$ time is available for uplink transmission. The case considering computing and feedback time will be studied in future work.

Denote the set of users which choose to offload by $\mathcal{N}$, $|\mathcal{N}|=N \in[0, K]$. The users adopt NOMA for offloading, and it is assumed that the users' messages are decoded in an increasing order of their indices. ${ }^{1}$ Denote the transmit power for the $i$ th user by $P_{i}$. Due to successive interference cancellation, the maximum achievable transmission rate of user $i$ becomes [17]

$$
r_{\mathrm{O}, i}=B(1-a) \log _{2}\left(1+\frac{P_{i} h_{i}}{\sum_{k=i+1}^{N} P_{k} h_{k}+N_{0}}\right) .
$$

where $B$ and $N_{0}$ represent the channel bandwidth and the noise power, respectively.

\section{Problem Formulation}

This paper aims to maximize the total computation rate of all users. According to (2) and (4), the computation rates of the users depend on the time allocation of WPT, on the computing mode selection and on the computing frequency or transmit power control for locally computing and for offloading users respectively. The sum computation rate maximization problem can be formulated as

$$
\begin{array}{ll} 
& \text { P1 }: \operatorname{maximize}_{a, P_{i} f_{i}, t_{i}, \mathcal{N}} \sum_{i \in \mathcal{N}} r_{\mathrm{O}, i}+\sum_{i \in \mathcal{K} \backslash \mathcal{N}} r_{\mathrm{L}, i} \\
\text { s.t. } & \mathrm{C} 1: \Gamma_{i} f_{i}^{3} t_{i} \leq \mu P h_{i} a T, \forall i \in \mathcal{K} \backslash \mathcal{N} \\
& \mathrm{C} 2: P_{i}(1-a) \leq \mu P h_{i} a, \forall i \in \mathcal{N},
\end{array}
$$

where $(5 b)$ means that the energy consumption of each user adopting local computing cannot exceed the harvested energy, while (5c) means that the transmit energy of the offloading users cannot exceed the harvested one. We do not consider a potential maximum transmit power constraint, since such a constraint is not likely to be reached in a WPT system, owing to the relatively low received energy.

\section{BINARY OFFLOADING WITH NOMA}

Problem (5) is clearly non-convex owing to the binary mode selection of each user and the multiplicative terms in both the objective function and constraints. However, once $a$ is given,

\footnotetext{
${ }^{1}$ As will be shown in the following sections, the decoding order does not affect the sum rate. For convenience, we use this decoding order here.
}

the optimization of $f_{i}$ and $t_{i}$ can be handled independently for each user. Then, as shown in [13, Lemma 1], the maximum $r_{\mathrm{L}, i}$ is achieved when $t_{i}=T$ and $f_{i}=\left(E_{\mathrm{H}, i} /\left(\Gamma_{i} T\right)\right)^{\frac{1}{3}}$.

Consequently, we have

$$
r_{\mathrm{L}, i}=\frac{\left(\mu P h_{i} a\right)^{\frac{1}{3}}}{\Gamma_{i}^{\frac{1}{3}} \phi_{i}} .
$$

Then, problem (5) can be equivalently reformulated as

$$
\begin{aligned}
& \text { P2 } \underset{a, P_{i}, \mathcal{N}}{\operatorname{maximize}} \sum_{i \in \mathcal{N}} r_{\mathrm{O}, i}+\sum_{i \in \mathcal{K} \backslash \mathcal{N}} \frac{\left(\mu P h_{i} a\right)^{\frac{1}{3}}}{\Gamma_{i}^{\frac{1}{3}} \phi_{i}} \\
& \text { s.t. } \quad \mathrm{C} 2: P_{i}(1-a) \leq \mu P h_{i} a, \forall i \in \mathcal{N} .
\end{aligned}
$$

To solve problem (7), we need to determine $\mathcal{N}, a$ and $P_{i}, \forall i \in \mathcal{N}$. The main difficulty comes from the combinatorial part $\mathcal{N}$, and its coupling with $a$ and $P_{i}$. Therefore, we divide the problem into two parts. First we assume that $\mathcal{N}$ is given, and consider the optimization of $a$ and $P_{i}$ in subsection A. We get back to determine $\mathcal{N}$ in subsection $\mathrm{B}$.

\section{A. Optimal Resource Allocation under Given $\mathcal{N}$}

Given $N$, the sum rate of these offloading users is

$$
\begin{aligned}
r_{\text {sum }}^{\mathrm{O}} & =B(1-a) \sum_{i \in \mathcal{N}} \log _{2}\left(1+\frac{P_{i} h_{i}}{\sum_{k=i+1}^{N} P_{k} h_{k}+N_{0}}\right) \\
& =B(1-a) \log _{2}\left(\frac{\sum_{i=1}^{N} P_{i} h_{i}+N_{0}}{N_{0}}\right),
\end{aligned}
$$

where the second equality holds because the sum rate expression forms a telescopic series.

According to (8), the sum rate is a function of $\sum_{i=1}^{N} P_{i} h_{i}$, which includes all offloading users, and is clearly independent of the ordering. Therefore, it can be concluded that the sum rate under NOMA is independent of the decoding order [6]. Nonetheless, the decoding order does affect the individual rate distribution, and thus, the fairness of the system. The minimum rate under maximum sum rate constraint is maximized when the users are decoded in the order of decreasing channel gains, that is, when the user with the weakest channel is decoded last, without interference [6].

Theorem 1: Consider Optimization problem (7), the sum rate is maximized when each offloading user transmits with maximum power satisfying the energy constraint, i.e.,

$$
P_{i}=\frac{\mu a P h_{i}}{1-a}, \forall i \in \mathcal{N} \text {. }
$$

Proof: The sum rate is monotonically increasing with $P_{i}, \forall i \in \mathcal{N}$. As $P_{i}$ 's are independent from each other, each user should transmit with maximum power. Considering the energy constraint $\mathrm{C} 2$, we can obtain $P_{i}=\mu a P h_{i} /(1-a)$.

Based on Theorem 1, the sum rate of the offloading users is given by

$$
\begin{aligned}
r_{\text {sum }}^{\mathrm{O}} & =B(1-a) \log _{2}\left(\frac{\frac{\mu a P \sum_{i=1}^{N} h_{i}^{2}}{1-a}+N_{0}}{N_{0}}\right) \\
& =B(1-a) \log _{2}\left(1+\frac{\rho a}{1-a}\right),
\end{aligned}
$$


where $\rho=\mu P \sum_{i=1}^{N} h_{i}^{2} / N_{0}$.

Let us now consider the allocation of time for wireless power transfer. Trivial algebra shows that $r_{\text {sum }}^{\mathrm{O}}$ is a concave function of $a$.

Let us consider the users that perform local processing. Their sum rate is the function of $a^{\frac{1}{3}}$, i.e., it is a concave function of $a$ as well. Since the sum of two concave functions is still concave, the overall sum rate maximization problem is concave and there exists a unique value of $a$, which maximizes the sum rate. Denote the derivative of the overall sum rate as $g(a)$, which is

$$
\begin{aligned}
g(a)= & B\left(\frac{(1-a)(\rho-1)}{\ln 2[(\rho-1) a+1]}-\log _{2}\left(\frac{(\rho-1) a+1}{1-a}\right)+\frac{1}{\ln 2}\right) \\
& +\frac{1}{3} a^{-\frac{2}{3}} \sum_{i \in \mathcal{K} \backslash \mathcal{N}} \frac{\left(\mu P h_{i}\right)^{\frac{1}{3}}}{\Gamma_{i}^{\frac{1}{3}} \phi_{i}} .
\end{aligned}
$$

The maximizer is achieved at the root of $g(a)$, i.e., $g(a)=0$. Even though there exists no closed form for the root, it can be obtained using bisection method. A possible implementation is summarized in Algorithm 1.

Once the optimal value of $a$ is found, the transmission power $P_{i}$ is defined by (9), while the offloading and local processing rates can be obtained from (4) and (6), respectively. Then, the resulting sum computation rate is given by $R_{\text {sum }}=\sum_{i \in \mathcal{N}} r_{\mathrm{O}, i}+\sum_{i \in \mathcal{K} \backslash \mathcal{N}} r_{\mathrm{L}, i}$.

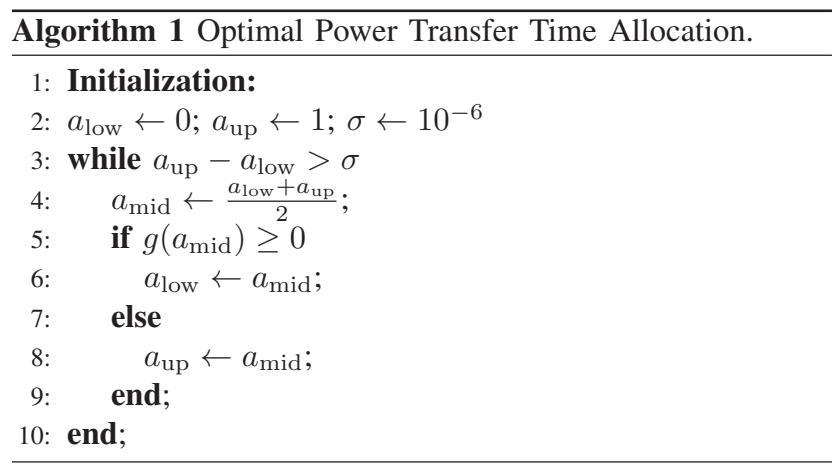

\section{B. Mode Selection Based on Channel Gains}

Now we consider how to choose the nodes to operate in offloading. To begin with, we show the following theorem, based on which we will propose the approach for mode selection.

Theorem 2: Consider Optimization problem (7), under binary offloading and NOMA based uplink transmission, the optimal set of offloading users $\mathcal{N}$, the energy transfer time $a$ and the maximum sum rate is independent from the NOMA decoding order.

Proof: The overall sum rate includes the sum rate from the offloading users and that from the local computing users. The sum rate of the locally computing users is not affected by the NOMA decoding. We have shown that the sum rate for the offloading users is independent of the decoding order. As a result, the overall sum rate under given $\mathcal{N}$ and any $a$ is independent from the decoding order, which also means that the optimal value of $a$ is not affected either. Finally, since this holds for any $\mathcal{N}$, the optimal mode selection is independent from the decoding order as well.

For the WPT assisted MEC network, the users suffer from double near-far effect during offloading [2], [3]. Previous results in [13] suggest that the optimal mode selection may have a threshold structure, where users with channel gains higher than this threshold perform offloading. Therefore, we propose a greedy user selection scheme to determine which user to offload. Specifically, users are first indexed in a descending order of their channel gains. Then, optimal resource allocation is performed considering $\mathcal{N}=\emptyset$ and $\mathcal{N}=\{1 \ldots n\}, n=1 \ldots K$. Finally, the configuration with the maximum sum rate is selected. The specific procedure is summarized in Algorithm 2.

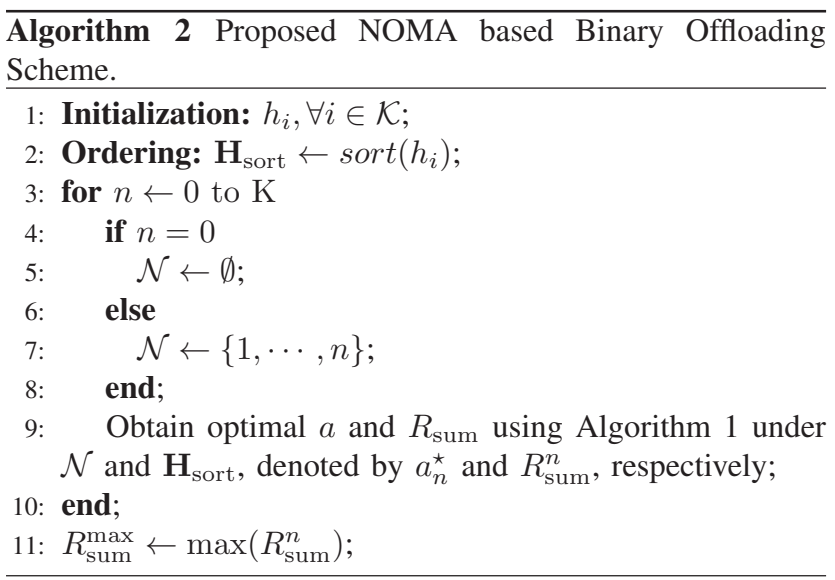

The complexity of Algorithm 2 is characterized by the following proposition:

Proposition 1: The complexity of the NOMA based binary offloading scheme is $O\left(K \log _{2}(K / \sigma)\right)$, where $\sigma$ is the threshold parameter for Algorithm 1.

Proof: First, ordering the users requires $O\left(K \log _{2}(K)\right)$ operations. Then, Algorithm 1 demands $O\left(\log _{2}(1 / \sigma)\right)$ operations, and it needs to run for each of the $K+1$ configurations of $\mathcal{N}$. The total complexity of the proposed scheme is then $O\left(K \log _{2}(K / \sigma)\right)$.

The proposition shows that the proposed scheme has low complexity, and thus, can be easily applied at the AP.

\section{Time Sharing for Better Fairness}

Theorem 2 guarantees same sum rates under different NOMA decoding orders. If only one decoding order is used during the transmission period $(1-a) T$, decoding the user with the strongest channel first maximizes the minimum rate of the offloading users. System fairness can be further improved by appropriately sharing the decoding orders in time domain [6]. That is, a decoding order is adopted only for a fraction of the duration $(1-a) T$. There exist $N$ ! decoding orders in total. Denote the ratio of time for the $m$ th ordering as $t_{m}$, $\sum_{m} t_{m}=1$. Let us denote by $M$ the matrix corresponding to all the decoding orders, and let $M(m, k)=i$ mean that user $i$ is the $k$ th decoded user in the $m$ th decoding order. 
Denote the throughput of user $i$ with time sharing by $\hat{r}_{i}$, which is given by

$\hat{r}_{i}=\sum_{m=1}^{N !} t_{m}\left(1-a^{\star}\right) \log _{2}\left(1+\frac{P_{i} h_{i}}{\sum_{l=k+1}^{N} P_{M(m, l)} h_{M(m, l)}+N_{0}}\right)$

where $a^{\star}$ denotes the optimal time allocation obtained from Algorithm 1. Substituting $P_{i}$ using (9) into the above equation, we have

$\hat{r}_{i}=\sum_{m=1}^{N !} t_{m}\left(1-a^{\star}\right) \log _{2}\left(1+\frac{\frac{\mu a^{\star} P h_{i}^{2}}{1-a^{\star}}}{\frac{\mu a^{\star} P \sum_{l=k+1}^{N} h_{M(m, l)}^{2}}{1-a^{\star}}+N_{0}}\right)$.

To achieve a better fairness, we aim to maximize the minimum rate, $R_{\text {min }}$, among the offloading users, by appropriately allocating the time for each decoding order. The considered optimization problem can be formulated as

$$
\begin{aligned}
& \text { P3 }: \underset{t_{m}}{\operatorname{maximize}} R_{\min } \\
\text { s.t. } & \mathrm{C} 3: \hat{r}_{i} \geq R_{\min }, \forall i \in \mathcal{N} .
\end{aligned}
$$

Since $\hat{r}_{i}$ is a linear combination of $t_{m}, m \in\{1, \cdots, N !\}$, P3 is clearly a linear programming problem, and can be efficiently solved using standard linear optimization tools, with polynomial complexity. Note that when $N$ is large, solving the linear programming over $N$ ! permutation space is still challenging. To address this, we can select the appropriate subset of $N$ ! permutation space for optimization to reduce the complexity [6].

To have a further understanding on the performance of the NOMA based binary offloading scheme, we will study a TDMA based binary offloading scheme as a benchmark in the next section.

\section{BASEline SChEme: BinARy OfFloAding With TDMA}

\section{A. Optimal Resource Allocation under Given $\mathcal{N}$}

When TDMA is used, the offloading time $(1-a) T$ has to be divided among the offloading users. Denote the offloading time for user $i$ as $\tau_{i} T$. Its corresponding rate is given by

$$
r_{i}=B \tau_{i} \log _{2}\left(1+\frac{P_{i} h_{i}}{N_{0}}\right) .
$$

Clearly, to maximize the rate, the maximum $P_{i}$ should be used. Considering the constraint of the harvested energy, we have $P_{i}=\mu a P h_{i} / \tau_{i}$. Substituting this into (15), we obtain

$$
r_{i}=B \tau_{i} \log _{2}\left(1+\frac{\eta a h_{i}^{2}}{\tau_{i}}\right),
$$

where $\eta=\mu P / N_{0}$. Accordingly, the offloading sum rate can be expressed as

$$
r_{\mathrm{sum}}^{\mathrm{O}}=B \sum_{i \in \mathcal{N}} \tau_{i} \log _{2}\left(1+\frac{\eta a h_{i}^{2}}{\tau_{i}}\right) .
$$

Before we present the theorem on the sum rate $r_{\text {sum }}^{\mathrm{O}}$, we introduce the following lemma:
Lemma 1: Consider

$$
R=\tau_{0} \log _{2}\left(1+\frac{x_{1}}{\tau_{0}}\right)+\left(1-\tau_{0}\right) \log _{2}\left(1+\frac{x_{2}}{1-\tau_{0}}\right),
$$

where $x_{1}$ and $x_{2}$ can be any positive value. $R$ is maximized when

$$
\tau_{0}=\frac{x_{1}}{x_{1}+x_{2}} .
$$

Proof: Simple algebra gives us that $\partial R / \partial \tau_{0}=0$ if $\tau_{0}=x_{1} /\left(x_{1}+x_{2}\right)$. Likewise, we have $\partial R / \partial \tau_{0}>0$ when $\tau_{0}<x_{1} /\left(x_{1}+x_{2}\right)$, and $\partial R / \partial \tau_{0}<0$ otherwise. Consequently, when (19) is satisfied, $R$ is maximized.

Theorem 3: The sum rate $r_{\text {sum }}^{\mathrm{O}}$ satisfies

$$
r_{\mathrm{sum}}^{\mathrm{O}} \leq B(1-a) \log _{2}\left(1+\frac{\rho a}{1-a}\right),
$$

where $\rho$ is defined at (10). The equality is achieved when $\tau_{i}$ is

$$
\tau_{i}=\frac{(1-a) h_{i}^{2}}{\sum_{k=1}^{N} h_{k}^{2}} .
$$

Proof: For notational simplicity, we consider a unit bandwidth $B$, so that $B$ can be removed from the rate expression.

We prove the theorem via mathematical induction. We introduce notation $H_{i}=\eta a h_{i}^{2}, i \in\{1, \ldots, N\}$.

The hypothesis for the induction is

$$
r_{n}^{\text {sum }} \leq\left(\sum_{i=1}^{n} \tau_{i}\right) \log _{2}\left(1+\frac{\sum_{i=1}^{n} H_{i}}{\sum_{i=1}^{n} \tau_{i}}\right),
$$

where $r_{n}^{\text {sum }}$ represents the sum rate of the first $n$ users, $n \in\{1, \ldots, N\}$. If the hypothesis holds for $n=N$, then the theorem is proved. Obviously, the hypothesis holds for $n=1$, since $r_{1}^{\text {sum }}=r_{1}=\tau_{1} \log _{2}\left(1+H_{1} / \tau_{1}\right)$.

Now let us prove that if the hypothesis holds for $n$, then it holds for $n+1$ as well.

$$
\begin{aligned}
& r_{n+1}^{\text {sum }} \\
& =r_{n}^{\text {sum }}+\tau_{n+1} \log _{2}\left(1+\frac{H_{n+1}}{\tau_{n+1}}\right) \\
& \leq\left(\sum_{i=1}^{n} \tau_{i}\right) \log _{2}\left(1+\frac{\sum_{i=1}^{n} H_{i}}{\sum_{i=1}^{n} \tau_{i}}\right)+\tau_{n+1} \log _{2}\left(1+\frac{H_{n+1}}{\tau_{n+1}}\right) \\
& =\left(\sum_{i=1}^{n+1} \tau_{i}\right)\left[\frac{\sum_{i=1}^{n} \tau_{i}}{\sum_{i=1}^{n+1} \tau_{i}} \log _{2}\left(1+\frac{\sum_{i=1}^{n} H_{i}}{\sum_{i=1}^{n+1} \tau_{i}} \frac{\sum_{i=1}^{n+1} \tau_{i}}{\sum_{i=1}^{n} \tau_{i}}\right)\right. \\
& \left.+\frac{\tau_{n+1}}{\sum_{i=1}^{n+1} \tau_{i}} \log _{2}\left(1+\frac{H_{n+1}}{\sum_{i=1}^{n+1} \tau_{i}} \frac{\sum_{i=1}^{n+1} \tau_{i}}{\tau_{n+1}}\right)\right]
\end{aligned}
$$

We introduce the auxiliary variable $\tau=\sum_{i=1}^{n} \tau_{i} / \sum_{i=1}^{n+1} \tau_{i}$. It also leads to $1-\tau=\tau_{n+1} / \sum_{i=1}^{n+1} \tau_{i}$. In addition, let $H_{1}^{\prime}=$ $\sum_{i=1}^{n} H_{i} / \sum_{i=1}^{n+1} \tau_{i}$ and $H_{2}^{\prime}=H_{n+1} / \sum_{i=1}^{n+1} \tau_{i}$.

Then we can write

$r_{n+1}^{\mathrm{sum}}$

$\leq\left(\sum_{i=1}^{n+1} \tau_{i}\right)\left[\tau \log _{2}\left(1+\frac{H_{1}^{\prime}}{\tau}\right)+(1-\tau) \log _{2}\left(1+\frac{H_{2}^{\prime}}{1-\tau}\right)\right]$.

According to Lemma 1 , when $\tau=H_{1}^{\prime} /\left(H_{1}^{\prime}+H_{2}^{\prime}\right)$, i.e., $\sum_{i=1}^{n} H_{i} / \sum_{i=1}^{n} \tau_{i}=H_{n+1} / \tau_{n+1}$, the expression 


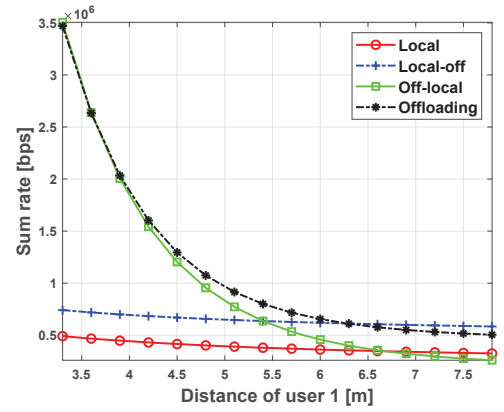

(a) Sum rate

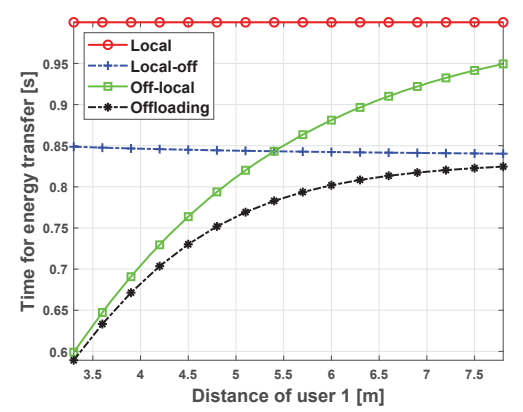

(b) Time for energy transfer

Fig. 2: Distance for user 1 varies; $d_{2}=5.4 \mathrm{~m} ; \phi=10$ cycles/bit.

in the bracket is maximized, and equals $\log _{2}(1+$ $\left.\sum_{i=1}^{n+1} H_{i} / \sum_{i=1}^{n+1} \tau_{i}\right)$. Consequently, $r_{n+1}^{\text {sum }}$ can be upper bounded as

$$
r_{n+1}^{\mathrm{sum}} \leq\left(\sum_{i=1}^{n+1} \tau_{i}\right) \log _{2}\left(1+\frac{\sum_{i=1}^{n+1} H_{i}}{\sum_{i=1}^{n+1} \tau_{i}}\right),
$$

which satisfies the hypothesis for $n+1$.

Finally, we consider the case of $N$ users. Due to $\sum_{i=1}^{N} \tau_{i}=$ $1-a$, we have

$$
\begin{aligned}
r_{N}^{\mathrm{sum}} & \leq\left(\sum_{i=1}^{N} \tau_{i}\right) \log _{2}\left(1+\frac{\sum_{i=1}^{L} H_{i}}{\sum_{i=1}^{N} \tau_{i}}\right) \\
& =(1-a) \log _{2}\left(1+\frac{\rho a}{1-a}\right)
\end{aligned}
$$

That is, the inequality of Theorem 3 is proved. Moreover, it is easy to conclude that the equality is achieved when $H_{1} / \tau_{0}=\cdots=H_{N} / \tau_{N}$. Correspondingly, we have $\tau_{i}=(1-a) H_{i} / \sum_{k=1}^{N} H_{k}=(1-a) h_{i}^{2} / \sum_{k=1}^{N} h_{k}^{2}, \forall i \in$ $\{1, \ldots, N\}$.

Corollary 1: Suppose (21) be satisfied, for given $\mathcal{N}$ and $a$, TDMA can achieve the same sum rate for the offloading users as NOMA.

Proof: When (21) is satisfied, $r_{\text {sum }}^{\mathrm{O}}$ is maximized, i.e., $r_{\text {sum }}^{\mathrm{O}}=B(1-a) \log _{2}(1+\rho a /(1-a))$. Comparing $r_{\text {sum }}^{\mathrm{O}}$ with the offloading part in (10), we can conclude that the same offloading rate is achieved for both NOMA and TDMA.

As a consequence of Corollary 1, the optimal $a$ should be the same for both NOMA and TDMA, and thus, Algorithm 1 can also be used to obtain the optimal $a$ for TDMA under given set of offloading users $\mathcal{N}$. The corresponding transmit power for user $i$ is given by

$$
P_{i}=\frac{a \mu P h_{i}}{\tau_{i}}=\frac{\mu P a \sum_{k=1}^{N} h_{k}^{2}}{(1-a) h_{i}}=\frac{C_{1}}{h_{i}},
$$

where $C_{1}=\mu P a \sum_{k=1}^{N} h_{k}^{2} /(1-a)$ is the same for all users.

\section{B. Binary Offloading Performance of TDMA}

The following corollary shows the perforamnce of the TDMA based binary offloading:

Corollary 2: Under binary offloading, TDMA can achieve the same sum rate as NOMA.
Proof: Due to Corollary 1, NOMA and TDMA achieves the same offloading rate for given $\mathcal{N}$ and $a$. Consequently, the optimal $a$ value and the maximum offloading rate is the same for the two access schemes. As the rate of the locally computing users is independent from the access scheme, the optimal mode selection and consequently the achievable sum rate has to be the same for NOMA and TDMA.

While binary offloading with NOMA and TDMA achieves the same sum rate, it can be seen that in TDMA users with weak channels are assigned little time for transmission according to (21). This means that under sum rate maximization, the achievable rate of these users, already suffering from the double near-far effect, is further reduced. This could lead to severe system unfairness. To avoid this unfairness, more time should be allocated to users with weak channels, which in turn decreases the achievable sum rate.

\section{Simulation Results}

In this section, simulations are conducted to evaluate the performance of the proposed NOMA-based binary offloading scheme. The simulator is implemented in Matlab, and the default simulation parameters follow the IoT scenarios in [13], [16]. The energy harvesting efficiency is set to $\mu=0.51$, and the computing efficiency coefficient is $\Gamma_{i}=10^{-24}$. The bandwidth is $B=5 \mathrm{MHz}$, while the receiver noise power is $N_{0}=10^{-10} \mathrm{~W}$. Without loss of generality, we normalize $T=1$. In addition, Rayleigh fading is assumed for small scale fading. The users are randomly distributed with a distance within 1 to $10 \mathrm{~m}$. The path-loss follows the free-space model $h_{i}=A_{d}\left(3 \times 10^{8} /\left(4 \pi f_{c} d_{i}\right)\right)^{d_{e}}$, where $d_{i}$ denotes the distance. $A_{d}=4.11, f_{c}=915 \mathrm{MHz}$ and $d_{e}=2.8$ represent the antenna gain, the carrier frequency, and the path loss exponent, respectively. The values of the transmit power $P$, the per bit computation cycles $\phi$ and the number of users $K$ are varied in the simulations, with default values of $P=40 \mathrm{dBm}$, $\phi=10$ cycles/bit and $K=10$. Unless mentioned explicitly, the obtained results are averaged over $10^{3}$ random trials. Recall that TDMA satisfying (21) can achieve the same sum rate as NOMA. Therefore, we do not show the sum rate of TDMA in the following simulation results, but evaluate its fairness only.

First, we would like to investigate how the channel gains affect the optimal mode selection, the time for energy transfer, 


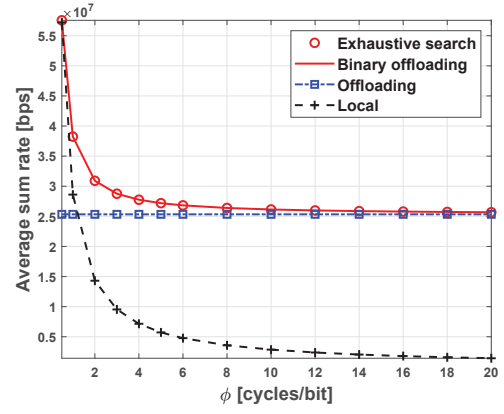

(a)

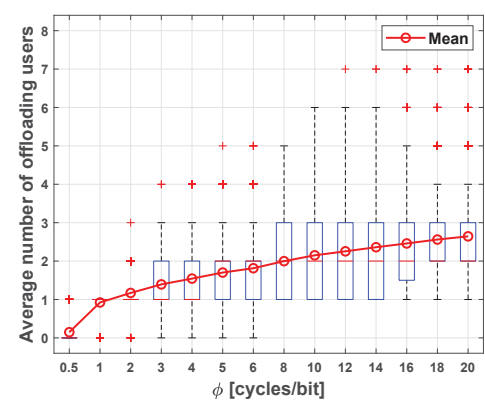

(b)

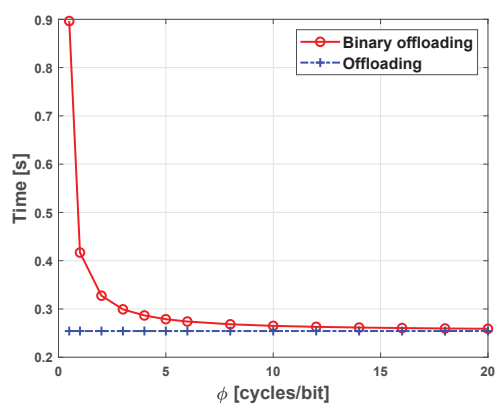

(c)

Fig. 3: Computation performance of Algorithm 2 versus $\phi$ under $K=10$ and $P=40 \mathrm{dBm}$ in terms of (a) average sum rate; (b) average number of offloading users; and (c) average time allocation.

and the achievable sum rate. Thus, we consider a simple scenario with two users, and the result is shown in Fig. 2. The distance of user 2 is fixed to be $d_{2}=5.4 \mathrm{~m}$, while that of user 1 varies. To remove the randomness of the channel qualities, small scale fading is not considered. There exist four cases of binary offloading: 1) both users process locally (denoted as Local); 2) user 1 processes locally, while user 2 offloads (denoted as Local-off); 3) user 2 offloads, while user 1 processes locally (denoted as Off-local); and 4) both users offload (denoted as Offloading). Fig. 2(a) shows the achieved sum rate. As the distance of user 1 increases, the optimal mode first changes from Off-local to Offloading, and then from Offloading to Local-off. This shift implies that the user with better channel has high gain of offloading (the Off-local and the Local-off curves cross each other when the two users are in the same distance from the AP). The stronger user can even force the weaker user to do local computation. According to Fig. 2(b), the optimal energy transfer time for Local is always one, as expected. As the distance for user 1 increases, the optimal time for energy transfer increases rapidly for Offlocal and Offloading, but declines slightly for Local-off. The former is probably because more energy transfer is required to compensate for the channel degradation, which causes double near-far effect for the offloading user, while the latter could be because the gain in information transfer outperforms the loss from local processing. Offloading has the lowest energy transfer time, since in this case none of the users would prefer energy transfer for the entire time frame.

Fig. 3 shows the computation performance of the MEC system versus $\phi$, the number of computation cycles required for processing one bit of raw data. In Fig. 3 (a), Exhaustive search gives the optimal results considering all possible user mode selections, while Binary offloading denotes the proposed NOMA-based scheme. Offloading and Local represent the results when all users are offloading and perform local computing, respectively. It is clear that Binary offloading achieves the same rate as Exhaustive search under any $\phi$ value, and thus, is optimal. Besides, as $\phi$ increases, the rate for Offloading remains unchanged, since changing $\phi$ does not affect the computation capabilities of offloading users. In contrast, both Binary offloading and Local decline with $\phi$. The latter is declining much faster, since all users are affected by $\phi$. In addition, Local is superior to Offloading only when $\phi$ is small, but Binary offloading always outperforms the other two schemes. The gain is significant at mediate $\phi$ values, and diminishes as $\phi$ becomes too large or small. Fig. 3(b) plots the corresponding number of offloading users using box plot. In the box plot, the box gives the first and third quartiles, while the line inside the box denotes the median value. The black line surrounding the box is the inner fence, which is the 1.5 interquartile range to the box. The points outside the inner fence are outliers. In addition, we also show the mean values. It can be seen that more users will offload when $\phi$ increases and local processing becomes less attractive. At large values of $\phi$, the number of offloading users is occasionally really high, but in average only a small portion of them is offloading, since the available per user transmission resources decrease quickly. Fig. 3(c) presents $a$, the related energy transfer time allocation. Local is not shown here, since there the entire time frame is used for energy transfer. As expected, the energy transfer time under Offloading remains unchanged. Meanwhile, the energy transfer time under Binary offloading declines with $\phi$, but is always larger than that under Offloading. Since offloading users prefer smaller energy transfer time, more offloading user will force the optimal $a$ towards smaller values.

Fig. 4 shows how the computation performance varies with the transmit power at the AP. We set $\phi=10$ cycles/bit, to consider a scenario when many users are offloading and the mode selection algorithm can be evaluated. Under this value, Offloading is close to Binary offloading, and is much better than Local, as we have already seen on Fig. 3(a). Our conclusions hold for other $\phi$ values as well, with higher gain of Binary offloading. According to Fig. 4(a), Binary offloading achieves the same performance as Exhaustive search, and outperforms the other two baseline schemes. The gap between Binary offloading and Offloading enlarges slightly when the transmit power increases. Meanwhile, Local is much worse than the other two, with significantly increasing gap. Fig. 4(b) evaluates the corresponding number of offloading users. Quite surprisingly, increasing the transmit power does not necessarily yield more offloading users. In fact, after a certain point, this could even lead to less offloading users. Overall, the 


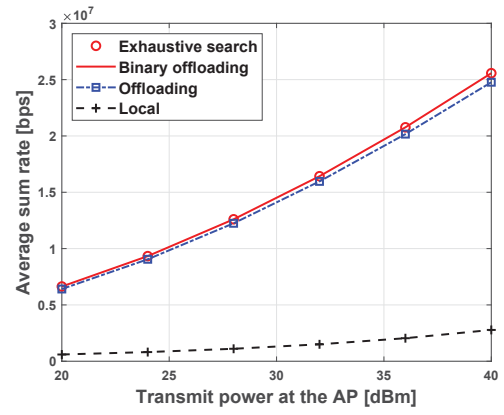

(a)

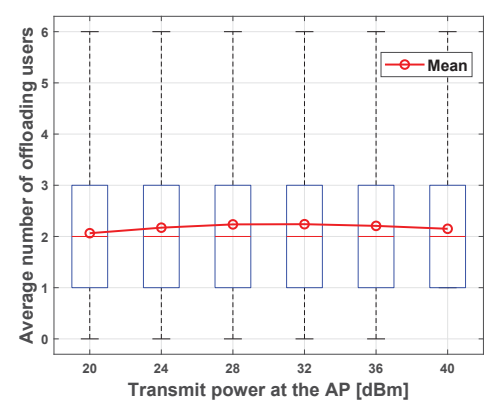

(b)

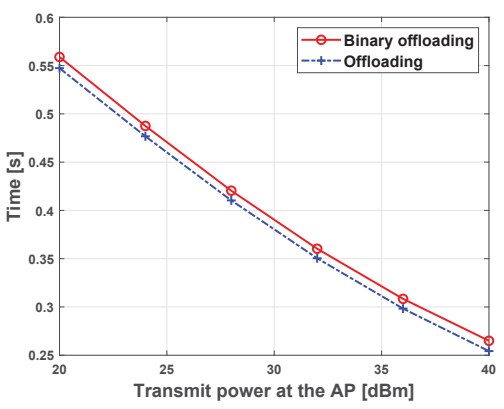

(c)

Fig. 4: Computation performance of Algorithm 2 versus transmit power at the AP under $K=10$ and $\phi=10 \mathrm{cycles} / \mathrm{bit}$ in terms of (a) average sum rate; (b) average number of offloading users; and (c) average time allocation.

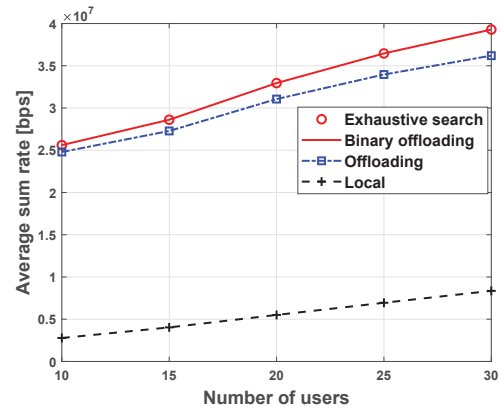

(a)

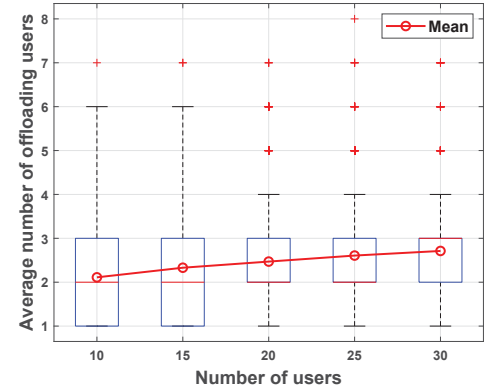

(b)

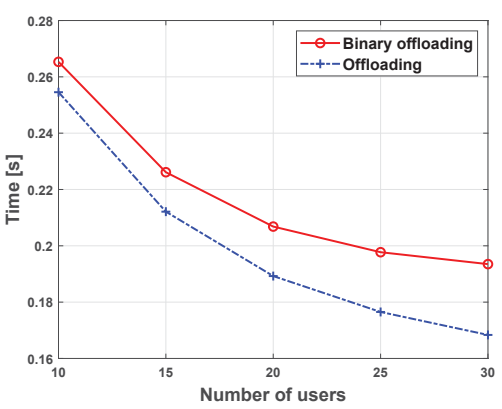

(c)

Fig. 5: Computation performance of Algorithm 2 versus number of users under $P=40 \mathrm{dBm}$ and $\phi=10 \mathrm{cycles} / \mathrm{bit}$ in terms of (a) average sum rate; (b) average number of offloading users; and (c) average time allocation.

changes are quite minor, which indicates that the AP power has very little effect on the number of offloading users. Fig. 4(c) plots the related energy transfer time allocation. As expected, the energy transfer time under Binary offloading is larger than that under Offloading. Moreover, both decline with the AP power. This is because $a$ decreases for the offloading users when the AP power increases and more energy is available.

Fig. 5 plots the variation of the computation performance with the number of users. According to Fig. 5(a), Binary offloading still achieves the same performance as Exhaustive search, and is better than the other two baseline schemes. Meanwhile, as expected, the rate increase under Local is linear. Fig. 5(b) presents the corresponding number of offloading users. Although the number of offloading users increases with the total number of users, the increment is quite minor. In particular, for $K=30$, less than three users on average are offloading. This is because the inter-user interference among the offloading users restricts the growth of the number of offloading users. We see as well that the randomness of the number of offloading users declines with increasing number of users, due to the decreased variance of the experienced channel quality. Fig. 5(c) shows the related time allocation. The optimal $a$ declines with the number of users. This could be explained by the fact that now the few offloading users have better channels, and require less energy harvesting time.

Finally, let us compare the different channel sharing schemes for binary offloading, i.e., TDMA, NOMA and NOMA-TS. As we know from Theorem 1, they all have the same optimal $a$ value, and they all achieve the same sum rate. In Fig. 6 we compare their fairness values, evaluated according to Jain's fairness index (JFI), i.e., $\mathcal{J}\left(x_{1}, \cdots, x_{n}\right)=\frac{\left(\sum_{i=1}^{n} x_{i}\right)^{2}}{n \cdot \sum_{i=1}^{n} x_{i}^{2}}$ [18]. Here NOMA denotes the case when users with better channels are decoded first, while NOMA-TS represents the case when time sharing is also adopted by NOMA. We evaluate fairness under increasing computation need, increasing AP transmit power and increasing number of users. It is clear that NOMA-TS outperforms both NOMA and TDMA in all considered scenarios, which shows the benefit of time sharing of the decoding orders. In addition, NOMA achieves better fairness than TDMA for most cases, which shows its superiority. One example for TDMA achieving better fairness than NOMA can be the situation when two users are offloading, and their channel gains are almost the same. In this case, the rates for the two users under TDMA are close, while those under NOMA are quite different. Therefore, TDMA achieves better fairness than NOMA.

Fig. 6(a) reflects the non-monotonic change of fairness as computations become heavier. As can be seen on Fig. 3(b), at small $\phi$ values all users perform local computation, which leads to relatively high fairness index. The fairness index then first falls as users start to offload, forcing a decreasing energy 


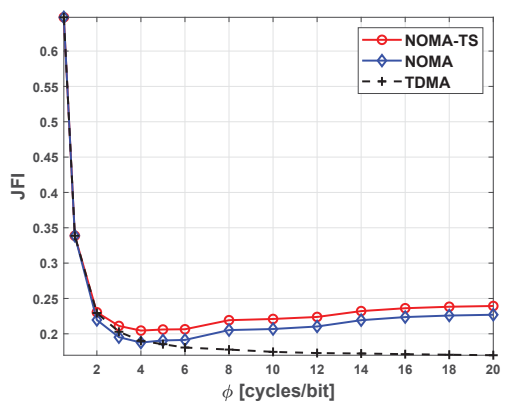

(a)

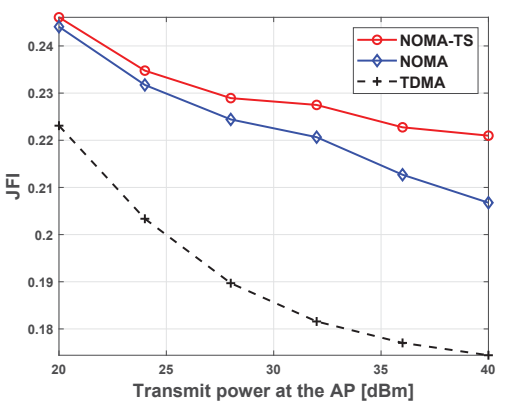

(b)

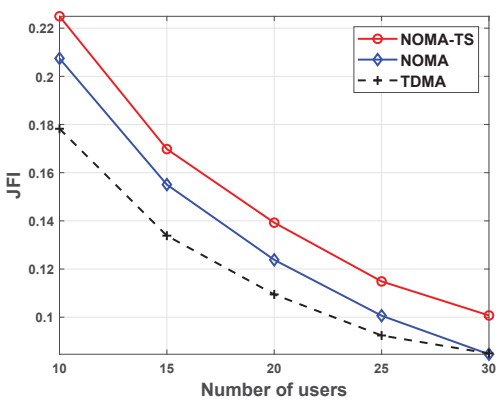

(c)

Fig. 6: JFI versus (a) $\phi$; (b) transmit power at the AP; and (c) total number of users.

transfer time, and thus unfairness between the offloading and the locally computing users, then it increases, as more users offload and achieve similar computation rate. Meanwhile, Fig. 6(b) shows that fairness decreases with increasing transmit power, since nearby users benefit significantly from the improved received power. Similarly, Fig. 6(c) reflects that fairness decreases as the number of users increases, and a few users with good channels benefit from offloading. For all cases the fairness values are not too high, since none of these schemes can improve the situation of locally computing users that have weak channels.

\section{CONCLUSION}

In this paper, we considered the problem of sum computation rate maximization in a WPT assisted MEC network with NOMA and with TDMA. The formulated problem requires a joint optimization of the individual computing mode selection and the time split between energy transfer and information transmission. The main difficulty of the system optimization comes from the combinatorial nature of the multiuser computing mode selection and its involved coupling with the time allocation. We proposed to decompose the original problem into two subproblems: 1) time and power allocation under given mode selection, and 2) mode selection. For the former, we provided an optimal solution, based on bisection search. For the latter, we proposed a greedy solution with threshold structure, based on the channel gains. We also gave the optimal time allocation for TDMA under given mode selection, and showed that TDMA can achieve the same sum rate as NOMA. Our numerical results demonstrated that for homogeneous networks the proposed binary offloading scheme achieves the same performance as exhaustive search, and thus, is optimal. The results show as well that binary offloading always outperform the two baseline algorithms, when all users offload or all users perform local computation, and NOMA can increase the fairness significantly, compared to a TDMA scheme with the same maximum sum rate. We also observed that the number of offloading users is quite small in all the considered scenarios, which relieves the implementation of NOMA.

\section{REFERENCES}

[1] S. Bi, C. K. Ho, and R. Zhang, "Wireless powered communication: opportunities and challenges," IEEE Commun. Mag., vol. 53, no. 4, pp. 117-125, Apr. 2015.
[2] H. Ju and R. Zhang, "Throughput maximization in wireless powered communication networks," IEEE Trans. Wireless Commun., vol. 13, no. 1 , pp. 418-428, Jan. 2014.

[3] L. Liu, R. Zhang, and K. Chua, "Multi-antenna wireless powered communication with energy beamforming," IEEE Trans. Commun., vol. 62, no. 12, pp. 4349-4361, Dec. 2014.

[4] M. Aboelwafa, et al., "Towards optimal resource allocation in wireless powered communication networks with non-orthogonal multiple access," Ad Hoc Networks, vol. 85, pp. 1-10, Mar. 2019.

[5] H. Chingoska, Z. Hadzi-Velkov, I. Nikoloska, and N. Zlatanov, "Resource allocation in wireless powered communication networks with non-orthogonal multiple access," IEEE Wireless Commun. Lett., vol. 5, no. 6, pp. 684-687, Dec. 2016.

[6] P. D. Diamantoulakis, K. N. Pappi, Z. Ding, and G. K. Karagiannidis, "Wireless-powered communications with non-orthogonal multiple access," IEEE Trans. Wireless Commun., vol. 15, no. 12, pp. 8422-8436, Dec. 2016.

[7] Y. Yuan and Z. Ding, "The application of non-orthogonal multiple access in wireless powered communication networks," in Proc IEEE SPAWC, Edinburgh, UK, Jul. 2016, pp. 1-5.

[8] Y. Mao, C. You, J. Zhang, K. Huang, and K. B. Letaief, "A survey on mobile edge computing: The communication perspective," IEEE Commun. Surv. Tuts., vol. 19, no. 4, pp. 2322-2358, 2017.

[9] P. Mach and Z. Becvar, "Mobile edge computing: A survey on architecture and computation offloading," IEEE Commun. Surv. Tuts., vol. 19, no. 3, pp. 1628-1656, 2017.

[10] C. You, K. Huang, and H. Chae, "Energy efficient mobile cloud computing powered by wireless energy transfer," IEEE J. Select. Areas Commun., vol. 34, no. 5, pp. 1757-1771, May. 2016.

[11] F. Wang, J. Xu, X. Wang, and S. Cui, "Joint offloading and computing optimization in wireless powered mobile-edge computing systems," in Proc IEEE ICC, Paris, France, May. 2017, pp. 1-6.

[12] F. Wang, "Computation rate maximization for wireless powered mobile edge computing," in Proc IEEE APCC, Perth, WA, Australia, Dec. 2017, pp. 1-6.

[13] S. Bi and Y. J. Zhang, "Computation rate maximization for wireless powered mobile-edge computing with binary computation offloading," IEEE Trans. Wireless Commun., vol. 17, no. 6, pp. 4177-4190, Jun. 2018.

[14] M. Zeng and V. Fodor, "Energy-efficient resource allocation for NOMAassisted mobile edge computing," in Proc IEEE PIMRC, Bologna, Italy, Sept. 2018, pp. 1794-1799.

[15] — "Energy minimization for delay constrained mobile edge computing with orthogonal and non-orthogonal multiple access," Ad Hoc Networks, submitted.

[16] Y. Wang, M. Sheng, X. Wang, L. Wang, and J. Li, "Mobile-edge computing: Partial computation offloading using dynamic voltage scaling," IEEE Trans. Commun., vol. 64, no. 10, pp. 4268-4282, Oct. 2016.

[17] M. Zeng and V. Fodor, "Sum-rate maximization under QoS constraint in MIMO-NOMA systems," in Proc IEEE WCNC, Barcelona, Spain, Apr. 2018, pp. 1-6.

[18] R. Jain, D. M. Chiu, and W. Hawe, "A quantitative measure of fairness and discrimination for resource allocation in shared computer system," DEC Research Report TR-301, 1984. 